\title{
Análise do domínio "Cognição" do Protocolo Individualized Music Therapy Assessment Profile (IMTAP)
}

\author{
Alexandra Monticeli \\ https://orcid.org/0000-0002-4968-8753 \\ Universidade Federal de Minas Gerais \\ xanda.rn@bol.com.br \\ Karina Marques \\ https://orcid.org/0000-0001-7568-0256 \\ Universidade Federal de Minas Gerais \\ psicoka@gmail.com
}

\author{
Ângela Pinheiro \\ https://orcid.org/0000-0002-5852-4320 \\ Universidade Federal de Minas Gerais \\ pinheiroamva@gmail.com
}

Rodrigo Vianna

https://orcid.org/0000-0002-0767-8338

Universidad del País Vasco

rvapsi@gmail.com

SCIENTIFIC ARTICLE

Submitted date: 11 sep 2020

Final approval date: 21 may 2021

Resumo: A maioria dos instrumentos avaliativos em Musicoterapia necessitam ter sua eficácia comprovada, direcionando as intervenções de forma condizente às necessidades do indivíduo. Portanto, investigou-se se uma série de atividades criadas para avaliar o domínio "Cognição" do protocolo IMTAP em relação a uma bateria formada pelos instrumentos psicológicos normalmente utilizados em avaliação cognitiva da leitura. Com base nas relações inferidas entre linguagem musical e a linguagem escrita e nos estudos sobre a intervenção musical na dislexia, explorou-se a possibilidade de que as crianças identificadas em risco de dislexia pudessem apresentar algum comprometimento no domínio "Cognição" do IMTAP, domínio que avalia o maior número de constructos similares aos itens avaliados nos instrumentos psicológicos. As expectativas do estudo, não foram confirmadas. As correlações de Pearson, embora tenham sido significativas, foram muito baixas, sugerindo que os constructos avaliados pelo IMTAP são diferentes dos construtos dos outros testes utilizados.

Palavras-chave: Musicoterapia; Análise; IMTAP; Transtornos específicos de aprendizagem; Música.

TITLE: ANALYSIS OF THE “COGNITION” DOMAIN OF THE INDIVIDUALIZED MUSIC THERAPY ASSESSMENT PROFILE (IMTAP) PROTOCOL

Abstract: Most assessment instruments in Music Therapy need to have their effectiveness proven, directing interventions in a manner consistent with the needs of individuals. Based on the relations inferred between musical language and written language, and studies on musical intervention in dyslexia, it could be hypothesized that children identified at risk of dyslexia could present some impairment in the "Cognition" domain of IMTAP, which evaluates the largest number of constructs similar to the items evaluated in psychological instruments. Therefore, this paper investigated whether a series of activities created to assess the "Cognition" domain of the IMTAP protocol was related to a battery encompassed by psychological instruments normally used in cognitive assessment of reading. The expectations of the study were not confirmed. Pearson's correlations, although significant, were very low, suggesting that the constructs evaluated by IMTAP are different from the constructs of the other tests used.

Keywords: Music therapy; Analysis; IMTAP; Specific learning disorders; Music. 


\section{Análise do domínio "Cognição" do Protocolo Individualized Music Therapy Assessment Profile (IMTAP)}

Alexandra Monticeli, Universidade Federal de Minas Gerais, xanda.rn@bol.com.br Ângela Pinheiro, Universidade Federal de Minas Gerais, pinheiroamva@gmail.com Karina Marques, Universidade Federal de Minas Gerais, piscoka@gmail.com Rodrigo Vianna, Universidad del País Vasco, rvapsi@gmail.com

\section{Introdução}

A fase escolar é de suma importância para o desenvolvimento de uma criança tanto em aspectos cognitivos quanto nos sociais e psicológicos. É também neste momento que problemas de aprendizagem tendem a ser notados por pais, cuidadores e professores, uma vez que se trata de uma fase de desenvolvimento e aprendizagem de novas habilidades.

Uma das publicações mais consultadas sobre o tema dos transtornos mentais é o Manual Diagnóstico e Estatístico dos Transtornos Mentais, já em sua 5a edição (DSM-5). Esse manual exerce grande influência na área da saúde e da educação por reunir dados e informações sobre os transtornos mentais, especialmente sobre o Transtorno Específico de Aprendizagem (TEAP), os quais foram produzidos a partir de evidências científicas alcançadas por diversos campos do conhecimento como a neurociência, a ciência cognitiva e as observações clínicas no campo das ciências da saúde.

De acordo com o DSM-5, o conceito TEAp inclui as seguintes condições de transtornos: Dislexia, Dificuldades de Compreensão de Leitura, Dificuldades de Soletração e/ou com a Expressão Escrita e a Discalculia. Esses transtornos têm em comum muitas características, tais como procedência biológica e impacto no neurodesenvolvimento da criança e em sua aprendizagem da leitura, na escrita e/ou cálculo matemático, habilidades desenvolvidas na fase escolar. Diferentemente da linguagem falada, essas habilidades devem ser ensinadas e aprendidas explicitamente.

Vale lembrar que o TEAp é uma condição diferente da chamada "dificuldade de aprendizagem", que surge de carências ambientais que impactam na aprendizagem da criança de forma geral. Ao contrário, esse transtorno afeta funções cognitivas específicas, como as habilidades de ler e escrever e/ou de calcular, como enfatizam Pinheiro e Scliar-Cabral (2017).

Para se chegar a um diagnóstico, o DSM-5 estabelece uma combinação de quatro critérios ( $A, B, C$ e $D)$ e um especificador da área do comprometimento para traçar o perfil do indivíduo. É importante que haja também uma análise do histórico de desenvolvimento da criança, considerando condições médicas, fisiológicas, familiares e escolares. Uma vez feita essa análise, deve-se buscar a tipificação sob a qual os domínios prejudicados se encontram. Quando esse domínio é a leitura, foco do presente artigo, "Dislexia" é o nome 
alternativo para o "Transtorno Específico de Aprendizagem com prejuízo na leitura", que se caracteriza especialmente por uma lentidão em reconhecer e ler palavras em voz alta e em altas taxas de erros em tais tentativas.

Além das "dificuldades com palavras", comumente a dislexia é associada a dificuldades de concentração, memória de curto prazo, organização e sequenciação de itens, conforme mencionado no site DislexiaBrasil (dislexiabrasil.com.br). Existe também na literatura sugestões vindas dos estudos de intervenção musical de que os disléxicos possam apresentar dificuldade com o ritmo e processamento auditivo, o que nos remete à relação entre a linguagem musical e a linguagem escrita (Montello e Coons 1998; Schlaug 2005; O'Brien 2004; Cogo-Moreira 2009; Lundetræ et al. 2017). O interesse em pesquisar tais relações, especialmente entre habilidades musicais e as habilidades de leitura e escrita, vem crescendo nos últimos anos (Gordon et al. 2015). Já é possível encontrar estudos onde vemos relatos de treinamento musicais relacionados com melhoras no âmbito acadêmico (Fischer 2001), bem como pesquisas buscando entender os mecanismos que estão envolvidos na ligação entre habilidades musicais e a linguagem oral/escrita (Peretz 2015).

Segundo Schlaug (2005), tocar um instrumento requer uma série de habilidades, incluindo ler um sistema simbólico e traduzi-lo em uma atividade motora sequencial e bimanual. Para o autor, é nesta premissa que a intervenção musical está baseada - na ideia de que aprender a ler pode ser comparada às atividades de ler partitura e de tocar um instrumento, uma vez que ambas exigirem coordenação dos músculos oculares para se seguir uma linha de leitura (no caso de instrumentos melódicos) ou uma leitura simultânea, onde mais de uma linha melódica é tocada ao mesmo tempo (no caso de instrumentos harmônicos). É necessário desenvolver uma orientação espacial para se seguir a partitura e uma interpretação simbólica para reproduzir o que nela está escrito, podendo assim ser comparável com fazer uma leitura e interpretá-la. Todas estas habilidades do fazer musical podem ser catalisadoras no desenvolvimento da leitura e da escrita, por envolverem respostas motoras e cognitivas distribuídas pelo cérebro. A música possui a capacidade de agir em todas as áreas cerebrais (Schlaug 2005), o que abre a possiblidade de que seu processamento e o da linguagem se sobreponham.

Nessa direção, O’Brien (2004), em um estudo conduzido com uma professora de música, relata que a professora percebeu que as dificuldades de processamento sonoro, o déficit auditivo, o raciocínio lento e a confusão com as notas de seus alunos se assemelhavam aos problemas encontrados por eles na leitura e na escrita. No caso de dificuldades em seguir uma linha de raciocínio e em construir um planejamento, tendem a ser acompanhadas por limitações equivalentes em se seguir uma sequência de ações motoras e de respiração, para executar o que está escrito em uma partitura (ao realizar solfejos ou cantar uma música).

Cogo-Moreira (2009), enfocando a interação entre aprendizagem musical e o desenvolvimento das habilidades de leitura e de escrita, considera que o que explica tal interação é a chamada transferência (Barnett et al. 2002; Benson et al. 1997), ou seja, a capacidade que o cérebro tem de transferir algo aprendido em um contexto para se utilizar no desenvolvimento em outro contexto. Um exemplo claro disso ocorre quando um estudante de piano desenvolve melhor coordenação motora fina em decorrência de sua prática. Outro exemplo de transferência ocorre na fala nos domínios da prosódia (Thompson et al. 2004), da discriminação da altura na fala (Moreno et al. 2009) e na memória verbal (Kilgour et al. 2000). Claramente o quesito mais indicado como responsável por transferências favoráveis concentra-se no contorno geral da altura da fala e das sequências de sons (Foxton et al. 2003). 
Em 2017, Lundetræ et al. verificaram como o ritmo poderia influenciar as habilidades de leitura de palavras e de ortografia de 479 crianças de seis anos que estavam na primeira série. Elas foram testadas assim que iniciaram o ano letivo, realizando uma atividade rítmica com tambores visualizados em um tablet. De acordo com o ritmo que lhes era solicitado as crianças deviam bater nos tambores que apareciam na tela. A análise do desempenho de leitura e de ortografia dos participantes ao longo do ano, indicou que aqueles que atingiram menos de $20 \%$ de eficácia na tarefa rítmica tiveram também desempenhos mais baixos em tais habilidades. Sendo assim, a análise rítmica se mostrou uma boa forma de predizer o desempenho dos alunos.

Com base nas relações inferidas entre aprendizagem musical e o desenvolvimento de habilidades, especialmente as de linguagem seja ela oral ou escrita, muitos países, inclusive o Brasil, já incluíram a educação musical como matéria obrigatória da educação básica (Cogo-Moreira et al. 2009). Muito tempo depois de países como os Estados Unidos da América tornarem obrigatório o ensino de música nos primeiros anos escolares e eletivo nos últimos anos de colegial, é que o Brasil, em 2008, sancionou a lei 11.769, estabelecendo a obrigatoriedade do ensino de música nas escolas de educação básica do nosso país.

\subsection{Intervenção musical na dislexia}

Forgeard (2008) estudou o impacto da Musicoterapia na leitura de cinco crianças disléxicas com nove anos de idade (Grupo experimental) e as comparou com dez crianças da mesma idade, mas com capacidades normais de leitura (Grupo controle). No primeiro grupo, as crianças receberam atendimentos individuais em música. No outro, o controle, nenhum apoio musical foi oferecido, sendo que das dez crianças, cinco já haviam participado ou ainda participavam de atividades musicais (como grupos onde tocavam instrumentos) por mais de um ano. Foi encontrado que os disléxicos que receberam suporte musical, em relação ao grupo controle, melhoraram o ritmo, o processamento auditivo durante uma leitura e a consciência fonológica. Porém, os autores não descrevem como esta melhora foi medida, apenas citando tais dados em suas conclusões.

Em outro estudo, Jaarsma et al. (1998) investigaram a dificuldade na compreensão da notação musical na dislexia e se o equivalente dessa limitação poderia ser observado na linguagem escrita na mesma condição. Cinco crianças diagnosticadas com dislexia e quatro que não apresentavam transtorno algum, foram submetidas a um programa de intervenção em notação musical (com duração de 15 semanas, com sessões uma vez por semana). Os disléxicos mostraram problemas na representação de tempo e de ritmo e na sequência de notas, fato este que pôde ser equiparado com as dificuldades no processamento fonológico na leitura e com as dificuldades de planejamento da escrita. Além disso, em comparação às crianças típicas, os disléxicos se mostraram menos preocupados com as posições exatas das notas na pauta.

Em 2016, Habib conduziu um estudo onde foi aplicado o chamado CMT (Cognitive-Musical Training, Treinamento Cognitivo-Musical, em tradução livre). O CMT é um método que foi desenvolvido por fonoaudiólogos (Shaywitz apud Habib et al. 2016) com base em princípios amplamente reconhecidos de intervenção, com progressão sistemática e coerente e dirigidos a objetivos ao longo de uma estrutura hierárquica. $O$ autor alega que mesmo sendo um método da fonoaudiologia, vários exercícios foram construídos para abarcar dimensões e componentes da música (altura, duração, andamento, pulsação e ritmo), visando desenvolver tanto a percepção quanto a produção de tais elementos. Compreendem ainda componentes sensoriais e motores, que envolvem a criança em processos de transcodificação de uma modalidade para outra, como ao tocar um instrumento musical em sincronia com uma sequência musical ouvida, tocar ao instrumento uma notação escrita de um ritmo e aprender a tocar pequenas melodias em 
um instrumento (ler e reproduzir no instrumento o que está escrito na notação musical). O CMT foi aplicado em doze crianças diagnosticadas previamente com dislexia, que foram divididas aleatoriamente em três grupos com quatro crianças cada. Todas participaram das três sessões de treinamento, que foram: i) exercício musicais ministrados por um fonoaudiólogo; ii) educação musical com um professor de piano e iii) percussão corporal rítmica, com um fisioterapeuta. O procedimento foi aplicado por três dias, seis horas diárias, totalizando 18 horas de aplicação, em sessões de 45 minutos. Habib conclui que o aprendizado musical pode ser uma ferramenta ideal para se lidar com crianças com dislexia, uma vez que a música contém padrões e desperta áreas cerebrais que não são atingidas por outras terapias, como a melhora da percepção auditiva e da discriminação de movimentos sonoros e suas alturas correspondentes.

Como se pode ver são escassos os estudos na área. Além disso, mesmo que alguns tenham sido realizados dentro de um delineamento de ensaios clínicos, com grupo de intervenção e de controle, as amostras utilizadas são muito pequenas e, no que se refere à metodologia, não há o emprego de medidas pré e pósteste. Adicionalmente, em muitos estudos, a descrição dos procedimentos de treinamento musical é superficial, uma vez que a abordagem ou método musical empregado na intervenção não é mencionado.

Outro complicador na área é que, em muitos estudos, a intervenção é feita por meio de técnicas da educação musical e não das de Musicoterapia. A educação musical tem como alvo o ensino/aprendizagem de um instrumento e/ou da teoria musical. Quando usada como meio de intervenção, o pressuposto é o de que as aulas de música por si só propiciam a estimulação para o desenvolvimento das habilidades intencionadas, mas não musicais, o que vai ao encontro de seu objetivo principal, já que seus métodos são desenvolvidos para este fim. A Musicoterapia, em contraste, se mune da música e de seus elementos para desenvolver habilidades biopsicossociais, buscando alcançar seus propósitos terapêuticos traçados que justificam a procura pelo tratamento.

Diante do exposto, vê-se que o estado atual da arte sobre os feitos da intervenção via música na dislexia, seja por meio da educação musical ou da Musicoterapia, tem ainda um longo caminho a percorrer.

\subsection{A Musicoterapia}

Ao contrário do senso comum, o objetivo da Musicoterapia não é o de ensinar música e sim o de buscar respostas musicais. Sendo conduzida como uma forma de expressão e de prazer, ela propicia o desenvolvimento de potenciais (prevenção) e a reeducação (intervenção) e o reestabelecimento de funções (reabilitação) para o alcance de uma melhor integração intra/interpessoal, proporcionado, assim, uma melhor qualidade de vida ao indivíduo (Federação Mundial de Musicoterapia 1996).

A Musicoterapia é uma ciência que se utiliza da música e de seus elementos visando alcançar uma saúde global do indivíduo. Utiliza-se do ritmo, da melodia e da harmonia, para alcançar finalidades específicas. Ao entendê-los e vivenciá-los, a pessoa começa a perceber, por exemplo, que existe um ritmo nos movimentos da fala e da leitura e, até mesmo, na ação de se escrever. A percepção da entonação melódica das palavras pode levar a uma leitura fluida e consciente (Pérez 2015). A repetição e o reforço positivo constantes em sua prática são elementos que podem auxiliar a pessoa a perceber e produzir tais movimentos através de uma condução musical.

As intervenções em Musicoterapia objetivam o desenvolvimento através de audição, recriação, improvisação e composição musical (Bruscia 2016). Esses procedimentos têm se mostrado uma forma eficaz 
de tratamento de muitas condições, incluindo as neuropsicológicas. Seus métodos e técnicas próprios podem alcançar uma melhora na qualidade de vida das pessoas (Loureiro e Rosário 2017), sendo seus efeitos benéficos perceptíveis nos níveis de funcionamento fisiológico, emocional e intelectual (Bruscia 2016).

Existem diversas abordagens musicoterápicas, como a Analítica, a Musicocentrada, a Musicopsicoterapia, a Comportamental e a Neurológica. As abordagens da Musicoterapia são determinadas de acordo com a especialidade do musicoterapeuta condutor do processo.

Neste trabalho, optou-se por utilizar a Musicoterapia Neurológica. Essa abordagem considera a música como um estímulo mediador capaz de proporcionar respostas não musicais referentes à cognição, comunicação, afetividade e ao processo sensório-motor, uma vez que a estrutura e os padrões musicais são elementos capazes de organizar, estimular e guiar a atenção, a percepção e o comportamento do indivíduo (Loureiro 2009 apud Loureiro e Rosário 2017; Thaut, McIntosh e Hoemberg 2015).

A Musicoterapia Neurológica se divide em vinte técnicas que têm a finalidade de promover a reabilitação através da relação do estímulo musical com funções cerebrais (Thaut 2008). Esse conjunto de técnicas é subdividido em quatro grupos: três técnicas de reabilitação sensório-motora, oito técnicas de reabilitação de fala e linguagem, sete técnicas de reabilitação cognitiva e duas técnicas de funções executivas. A escolha da técnica depende do objetivo do processo terapêutico de cada caso. O musicoterapeuta tem a liberdade para criar o caminho do tratamento de acordo com a demanda do paciente e das necessidades levantadas pelo próprio ou por sua família (Thaut e Hoemberg 2014).

\subsection{The Individualized Music Therapy Assessment Profile - IMTAP}

Com o avanço dos processos musicoterapêuticos, muitos profissionais passaram a ter dificuldade em encontrar uma forma de avaliar e quantificar o desenvolvimento global de seus pacientes tanto em aspectos físicos quanto emocionais, sociais e até mesmo cognitivos, inclusive, como forma de obter um marco do estado do paciente no início do tratamento, a fim de apreciar o estado do paciente no final do processo, baseado em intervenções sistemáticas planejadas, longitudinais e sequenciais. Nessa busca, frequentemente, tem-se recorrido a avaliações da área da Psicologia ou da Educação, que, por sua vez, podem gerar avaliações incompletas ou mesmo imprecisas por não contemplarem aspectos específicos da Musicoterapia (Gregory 2000). Assim, com o objetivo de se criar um protocolo próprio da Musicoterapia, em 2007, um grupo de pesquisadores ingleses conduzidos por Baxter desenvolveu o chamado The Individualized Music Therapy Assessment Profile, conhecido como IMTAP. Ele foi pensado para que houvesse uma forma de se obter uma descrição narrativa sobre as sessões ao longo do processo.

O IMTAP é um protocolo que avalia o estado em que o indivíduo se encontra nos seguintes em dez domínios. São eles: musicalidade, comunicação expressiva, comunicação receptiva/percepção auditiva, interação social, motricidade ampla, motricidade fina, motricidade oral, cognição, habilidade emocional e habilidade sensorial. Seus resultados traçam o perfil do indivíduo, enfatizando os seus pontos fortes e as áreas em que mostra maior dificuldade. Sua aplicação pode acontecer em apenas um encontro - caso apenas um domínio seja avaliado - ou em vários encontros, além de apreciação do caso por gravações ou vídeos. Por isso, o protocolo não é utilizado para fazer diagnósticos e nem para a comparação entre sujeitos, permitindo apenas avaliar a mesma pessoa em diferentes momentos, indicando suas áreas com maiores potenciais e com maiores dificuldades. Baxter et al. (2007) chamam atenção para a grande versatilidade de utilização desse protocolo no que diz respeito às faixas etárias das pessoas que podem ser submetidas a ele (crianças, 
adolescentes e adultos) e à sua recomendação de aplicação tanto em pessoas sem uma queixa determinada ou com distúrbios diagnosticados. Em ambas as situações, o IMTAP é utilizado para avaliação do desempenho do indivíduo no início e no final do período de submissão ao processo de Musicoterapia.

No Brasil, Silva et al. (2013) traduziram o IMTAP e proveram validade de conteúdo para toda a versão brasileira do instrumento e validade convergente para apenas um de seus domínios: "comunicação expressiva". Embora os autores se refiram a essa versão como "Escala IMTAP", neste trabalho optou-se pela a utilização do nome "Protocolo IMTAP", uma vez que seus parâmetros são melhor inseridos neste tipo de denominação. (Coluci et al. 2015).

Neste trabalho, nos focamos no domínio "cognição" do IMTAP. Nele, encontram-se os subdomínios "memória" e "atenção", que podem ser úteis em avaliações em crianças com o TEAp. Os itens de tais subdomínios devem ser construídos de forma a testar a memória de curto e longo prazo, atenção focada e dividida, dentre outras habilidades, tais como a capacidade de tomada de decisão e de seguir instruções, essa última também deficitária em crianças com dislexia.

As atividades utilizadas no IMTAP não são prescritas ou precisam seguir uma abordagem específica. Cabe ao musicoterapeuta aplicar uma atividade que ele julgue pertinente para evidenciar aquele domínio buscado em seu paciente. Entretanto, neste trabalho, conforme apresentado em "Instumentos" no Método foi aplicada uma sequência de atividades pré-estabelecidas, respeitando um mesmo padrão, evitando, dessa forma, tendenciosidade e imprecisões.

\section{Justificativa}

Por mais que alguns estudos já tenham sido conduzidos aplicando a música a pessoas que apresentam TEAp, é possível notar que em sua maioria eles são pautados na área da educação musical. Não foi identificada pesquisa finalizada que vise demonstrar experimentalmente se a Musicoterapia pode vir a trazer alguma forma de auxílio no processo de melhora de tais transtornos. Há carência de instrumentos na área. A ferramenta de avaliação com potencial de atender às demandas da área da Musicoterapia no Brasil, o IMTAP, está validado apenas seu conteúdo, uma vez que, em termos de validade convergente, o único subdomínio submetido a estudos preliminares foi "comunicação expressiva". Conforme sugestão de Silva et al. (2013), é necessário que haja validação para todos os seus outros subdomínios.

Adicionalmente, considerando a relação entre a linguagem musical e a linguagem escrita e os possíveis efeitos da Musicoterapia na melhora de habilidades subjacentes à leitura (ex., ritmo e consciência fonológica), torna-se de interesse investigar a possibilidade do domínio "Cognição" do IMTAP ser útil para a identificação de crianças em risco de apresentar o TEAp de leitura, e consequentemente, dislexia. No entanto, antes de se conduzir um estudo de intervenção, é necessário que se tenha um instrumento confiável para se aferir as aptidões musicais antes e depois da intervenção na situação de pré e pós-teste, sendo este o objetivo desta pesquisa.

\section{Método}

\subsection{Amostra}

A amostra é composta por 30 alunos do 20 ano do ensino fundamental, provenientes de duas turmas de uma escola na cidade de Belo Horizonte. Ela foi distribuída igualmente entre os dois sexos, e com média de 
idade de 7 anos e 11 meses. O desvio padrão é de 4,5 meses, tendo o participante mais velho 8,75 anos e o mais novo, 7,08 anos. A escolha por alunos cursando o segundo ano escolar deveu-se à determinação do Ministério da Educação (MEC) de que o processo de alfabetização deve estar efetivado até o 20 ano do Ensino Fundamental, como descrito na Base Nacional Comum Curricular (BNCC), de acordo com a Resolução CNE/CP no 2 (2017).

\subsection{Instrumentos}

Os instrumentos consistiram da escala Cognição do IMTAP (Silva 2012), doravante referida como IMTAPCog, de medida para a avaliação da inteligência não verbal e de medidas para o levantamento de suspeita de risco de dificuldade de aprendizagem da leitura.

- IMTAP-Cog (Silva 2012)

Esse domínio do IMTAP foi escolhido devido ao seu potencial de propor atividades para testar diferentes funções cognitivas. Ele é dividido em seis subdomínios: Fundamentos, Tomada de decisão, Seguindo instruções, Recordação de curto prazo, Recordação de longo prazo e Acadêmicas (subdomínio não avaliado). "Fundamentos" constitui-se no primeiro subdomínio avaliado em cada um dos dez domínios do IMTAP. Ele elenca as características gerais e fundamentais do domínio que está sendo trabalhado (Silva 2012), enquanto os outros subdomínios são específicos ao domínio.

Assim, tomando como referência as técnicas da Musicoterapia Neurológica foram elaboradas atividades musicoterapêuticas para testar os construtos subjacentes a esses subdomínios. As técnicas utilizadas foram:

1. MACT - Treinamento de Controle da Atenção Musical (Musical Attention Control Training)

O MACT é uma técnica que envolve a aplicação de atividades musicais estruturadas para alcançarem respostas musicais específicas. Ele pode evocar respostas tanto de canções já compostas quanto de improvisadas. Os elementos musicais sinalizam as diferentes respostas esperadas com o objetivo de desenvolver os mais diversos tipos de atenção (concentrada, alternada e dividida).

2. APT - Treinamento de Percepção Auditiva (Auditory Perception Training)

A técnica APT estimula a percepção da audição e a integração sensorial. A forma de alcance deste objetivo é por meio de atividades que consistem em discriminação e identificação de diferentes componentes do som, como andamento, métrica, timbres, padrões, alturas etc. Para se alcançar êxito em atividades propostas com esta técnica, é preciso que haja integração de diferentes sentidos, como audição e visão ou tato e audição. Músicas que buscam imediatismos de resposta, onde a canção gera um tempo aberto para uma resposta rápida do paciente também são bons exemplos das aplicações da APT.

3. MEM - Treinamento de Memória Ecoica Musical (Musical Echoic Memory Training)

A Memória Ecoica faz parte do primeiro estágio do caminho da memória auditiva. Ela funciona como um registro da memória sensorial, retendo as informações imediatas do som. Ela também se encarrega de reconhecer um som anteriormente ouvido e trabalha na distinção e significação de tal som. 
O MEM busca estimular a recordação imediata de sons musicais, seja através de canto, execução instrumental ou por músicas gravadas. Podemos percebê-la em momentos onde uma música está sendo aprendida pela pessoa apenas pelo ato de se escutar e repetir a ação, quase ao mesmo tempo.

4. MSOT - Treinamento de Orientação Sensorial Musical (Musical Sensory Orientation Training)

Esta técnica se utiliza de música gravada ou ao vivo, para estimular o estado de atenção e vigilância, facilitando respostas que dizem respeito a orientações de tempo, lugar, espaço e sentimentos. Canções onde os nomes das pessoas são chamados para que elas realizem certos comandos, ou canções onde os comandos são dados de forma aleatória ou imprevisíveis, são grandes carros-chefes da MSOT.

Ao fim da avaliação uma medida é calculada, sendo possível que o paciente/participante atinja as seguintes proporções: $\mathrm{N}=$ Nunca $=0 \% ; \mathrm{R}=$ Raramente $=$ Abaixo de $50 \%$; I Inconsistente $=50-79 \%$ e $\mathrm{C}=$ Consistente $=80-100 \%$. Esta forma de se avaliar é denominada como Sistema NRIC que pode ser praticado de duas formas: por valor estimado ou por pontos. A forma estimada é mais utilizada em situações clínicas, nas quais os constructos de cada item são considerados de maneira mais abrangente, conforme os dados contidos nas descrições de cada letra do NRIC. Já o registro por pontos envolve uma contagem do número de oportunidades dadas para a execução da habilidade dividido pelo número de vezes em que a habilidade foi demonstrada, resultando em um registro mais preciso. $O$ registro por pontos foi o escolhido na presente pesquisa.

As atividades elaboradas e aplicadas foram:

1. Canção de abertura: "Bom dia", com espaço aberto para resposta.

Nesta atividade, se buscou avaliar a forma que cada criança expressa o seu sentimento em resposta ao cumprimento "Bom dia, bom dia" e à pergunta "como vai?" e a sua capacidade de:

$\checkmark$ dar uma resposta musical a um comando também musical;

$\checkmark$ manter um diálogo musical;

$\checkmark$ de atenção alternada e

$\checkmark$ memória de curto prazo.

Técnica utilizada - MACT.

2. A brincadeira Yapo: os alunos deveriam cantar e fazer os movimentos referentes à canção. Os movimentos foram ensinados no início da atividade. Buscou-se avaliar:

$\checkmark$ memória de curto prazo, para o aprendizado dos movimentos necessários para a execução daquela atividade e

$\checkmark$ atenção concentrada, para a manutenção de em uma atividade repetitiva.

Técnica utilizada - MEM e MSOT.

3. Atividade Bingo Sonoro: cartelas com imagens de objetos variados foram distribuídas entre as crianças, sentadas em um círculo no chão. Cada cartela continha seis objetos. Antes que o jogo tivesse início, foi assegurado que todas elas conheciam e podiam reproduzir os sons feitos pelos objetos apresentados (ex.: carro, campainha, descarga, chuva e buzina). A seguir, cada um dos estímulos sonoros foi apresentado de forma aleatória em uma caixa de som. As crianças deveriam marcar em sua cartela a imagem 
correspondente ao som executado. O exercício foi finalizado quando uma das cartelas estava completamente marcada.

Nesta atividade, se buscou avaliar:

$\checkmark$ memória de curto prazo, para o entendimento da instrução da atividade e para a manutenção do som na memória enquanto a criança vai a cartela para procurar a figura que o correspondia na cartela;

$\checkmark$ memória de longo prazo, para a recordação dos objetos emissores dos sons;

$\checkmark$ atenção concentrada durante a atividade, para não ser influenciada por elementos distratores ou pelas outras crianças e

$\checkmark$ atenção dividida e alternada, necessária para poder identificar o som emitido e compará-lo ao objeto que o emite.

Técnicas utilizadas - MEM, MACT e APT.

4. Atividade com as clavas: a música "Los Tres Golpecitos" ${ }^{1}$ foi colocada para tocar e um par de clavas foi dado a cada aluno. Seguindo o exemplo demonstrado, eles deveriam tocar no andamento e seguindo as marcações rítmicas sugeridas pela música. Nesta atividade, buscou-se avaliar:

$\checkmark$ a atenção concentrada da criança ao manter a atividade;

$\checkmark$ a atenção dividida, considerando que ela não deveria ser influenciada pelos outros alunos que estavam por perto e

$\checkmark$ sua memória de curto prazo, juntamente com sua capacidade de prever os comandos musicais que viriam.

Técnicas utilizadas - MACT e APT.

5. Atividade com instrumentos: uma música foi cantada, sendo que na letra foram inseridos alguns comandos para que as crianças os seguissem, executando com os instrumentos (como: alto, baixo, rápido, lento, em cima, em baixo, etc).

Nesta atividade, se buscou avaliar:

$\checkmark$ a atenção concentrada da criança ao manter a atividade;

$\checkmark$ a atenção dividida, considerando que ela não deveria ser influenciada pelos outros alunos que estavam por perto;

$\checkmark$ atenção alternada, para que ela pudesse entender o que deveria ser feito na atividade e buscasse meios para tal;

$\checkmark$ sua memória de curto prazo, juntamente com sua capacidade de prever os comandos musicais que viriam e

$\checkmark$ capacidade de seguir comandos e prever ações.

Técnicas utilizadas - MACT, MSOT e APT.

1 Los Tres Golpecitos - Carlos Gianni e Eduardo Segal Música para jugar - 1974. Disponível em: <https://www.youtube.com/watch?v=moE4R3daq70>. Acesso em: 29 de março de 2020. 
6. Fechamento: canção de despedida, que demonstra que aquele momento de atividades musicais chegou ao fim.

- Matrizes Progressivas Coloridas de Raven (MPCR - Raven 1949; Angelini et al. 1999)

O teste foi utilizado para a avaliação da inteligência não verbal. As MPCR avaliam a inteligência fluida em crianças de 5 a 11 anos e meio. Ele busca avaliar a capacidade de extrair significados de situações consideradas confusas, desenvolvendo novas formas de compreender, tomar decisões e de resolver problemas (Raven 1949). Pode ser aplicado tanto de forma individual quanto coletiva, sendo o limite máximo de 9 indivíduos por grupo. É composto por três séries com 12 itens que dependem da capacidade de completar padrões contínuos, de identificar figuras discretas e de entender analogias de padrão específico de raciocínio. O objetivo é verificar a aptidão do sujeito para apreender as relações entre as figuras. Para sua realização, o avaliando deve escolher uma opção dentre seis que melhor represente a lacuna faltante da gravura.

Medidas para o levantamento de suspeita de risco de dificuldade de aprendizagem da leitura:

- Subteste Dígitos da Escala de Inteligência Wechsler para Crianças - WISC-IV (Wechsler 2013)

A Escala de Inteligência Wechsler para Crianças tem por finalidade avaliar a capacidade intelectual de crianças. Ela foi desenvolvida levando-se em consideração a concepção da inteligência como uma entidade agregada e global, ou seja, capacidade do indivíduo em raciocinar, lidar e operar com propósito, racional e efetivamente com o seu meio ambiente.

- Bateria Psicológica para Avaliação da Atenção (BPA - Rueda et al. 2013)

Seu objetivo é realizar uma avaliação geral de atenção, tanto quanto uma avaliação individualizada de três tipos de atenção específicos: atenção concentrada ( $A C)$, atenção dividida ( $A D)$ e atenção alternada (AA). Ela é composta por três testes, cada um deles tendo como objetivo avaliar um dos tipos atencionais propostos. A análise dos três testes em conjunto fornece a medida de atenção geral (AG). O teste é constituído por vários estímulos abstratos, sendo que ora eles são estímulos-alvo, e ora estímulos-distratores. Em cada teste específico contêm 400 estímulos, distribuídos em 20 linhas com 20 estímulos cada. Destes 400, 120 são alvos e 280 são distratores.

- Teste de Repetição de Pseudopalavras para Crianças Brasileiras (BCPR - Santos e Bueno 2003)

O teste BCPR demanda a capacidade de memória fonológica, para codificar e manter na memória uma sequência fonológica nova para subsequente produção dos movimentos articulatórios da fala. Testa o estado da rota fonológica no processamento da fala, que inclui procedimentos de segmentação e síntese de fonemas. 0 teste é composto por 40 pseudopalavras, que são estímulos construídos respeitando a estrutura ortográfica de uma língua. Por isso podem ser repetidos, lidos e escritos, mas uma vez que são "inventados", não estão arquivados no léxico da língua. Portanto, não possuem significado (ex., "barita"). A cada pseudopalavra repetida corretamente, a criança deve marcar um ponto, sendo 40 pontos o total máximo.

- Tarefas de Nomeação Seriada Rápida (NSR - Denckla e Rudel 1974)

Para avaliar a velocidade de nomeação, serão aplicadas as tarefas de Nomeação Seriada Rápida (NSR) de Cores, Letras, Objetos e Números (originárias do Teste RAN de Denckla e Rudel 1974). Esse conjunto de 
tarefas de maneira geral testa a habilidade de processar símbolos visuais rapidamente, nomeando-os. Especificamente, avalia a precisão, a automaticidade, a sincronização e a integração de processos de natureza perceptual, responsáveis pelo reconhecimento de padrões visuais e lexicais, que permitem o acesso e a recuperação de informações semânticas, ortográficas e fonológicas bem como motoras que possibilitam a articulação da pronúncia dos estímulos. Todos estes processos são considerados fundamentais para o bom desempenho em leitura.

- Teste de Leitura e Compreensão de Sentenças (TELCS - Vilhena et al. 2016; Vilhena e Pinheiro 2020).

O Teste de Leitura e Compreensão de Sentenças é utilizado para se avaliar competências de leitura, vocabulário, sintaxe e compreensão, além de reconhecimento de palavras e velocidade de leitura, uma vez que a tarefa é cronometrada (Vilhena et al. 2016). O teste, de aplicação coletiva, foi elaborado para crianças do 20 ao 5 ano do ensino fundamental. 0 teste consiste em uma leitura silenciosa de sentenças que estão incompletas e que possuem uma lacuna para serem preenchidas pelas crianças.

Todos esses instrumentos foram correlacionados com o conjunto de subdomínios do domínio "Cognição" do IMTAP, assim com cada domínio separadamente.

\subsection{Procedimentos}

A pesquisa obteve aprovação ética pelo Comitê de Ética de Pesquisa (COEP - UFMG) sob o número de registro 10729619.7.0000.5149. Todos os alunos levaram para casa o TCLE em duas vias: uma via para a assinatura dos pais ou responsáveis e outra para a assinatura de consentimento do próprio participante.

A aplicação dos instrumentos ocorreu em dois encontros. Em cada um deles, os alunos foram divididos em dois grupos. Os participantes do primeiro grupo foram submetidos aos testes de aplicação coletiva; os participantes do segundo grupo, aos testes de aplicação individual. No encontro seguinte, a ordem foi invertida: os alunos submetidos aos testes coletivos fizeram os testes individuais e vice-versa.

Esse procedimento contou com uma extensa equipe de colaboradores, composta por alunos do curso de graduação em Psicologia, psicólogos e por musicoterapeutas. Foi precedido por treinamentos cuidadosos sobre a aplicação dos instrumentos, sendo que os testes psicológicos foram aplicados pela equipe de psicólogos e os de Musicoterapia, pelos profissionais dessa área.

\section{Resultados}

A análise dos dados foi feita utilizando o programa de estatísticas SPSS (IBM, Statistics SPSS, 25) em sua versão atualizada no ano de 2019. A Tabela 1 apresenta o desempenho da amostra $(n=30)$ no IMTAP-Cog. Vale lembrar que, uma vez que o IMTAP compara os resultados do indivíduo com os seus próprios em diferentes momentos da aplicação, não se tem uma média comparativa esperada.

Encontramos outliers nos resultados do IMTAP-Cog. Os seguintes cinco alunos tiveram um desempenho excepcionalmente inferior aos demais: AAG, AOG, ABG, MCS e TAM. Além desses, um aluno ficou limítrofe quanto a ser outlier na cauda inferior da distribuição dos escores padronizados: AJA. 
Tabela 1: Médias (pontos brutos) e desvios padrões para o domínio “Cognição” do IMTAP.

\begin{tabular}{cccc}
\hline IMTAP & Média & DP & $\begin{array}{c}\text { Pontuação } \\
\text { máxima possível }\end{array}$ \\
\hline Fundamentos & 8,13 & 1,63 & 10 \\
Tomada de Decisão & 15,23 & 2,22 & 18 \\
Seguindo Instruções & 10,27 & 0,98 & 11 \\
Recordação de Curto Prazo & 9,63 & 1,03 & 42 \\
Recordação de Longo Prazo & 35,4 & 4,25 &
\end{tabular}

A Tabela 2 mostra os resultados do Raven, considerando a amostra como um todo ou também separada por idade (7 e 8 anos). Para a idade de 7 anos encontrou-se média de 16,2 e desvio padrão (DP) de 5,3. E para 8 anos, uma média de 18,6 com desvio padrão de $(6,1)$.

Tabela 2: Estatísticas descritivas do Raven (escores brutos).

\begin{tabular}{cccc}
\hline & \multicolumn{3}{c}{ Raven } \\
\cline { 2 - 4 } & 7 anos & 8 anos & Geral \\
\hline Mínimo & 12,0 & 21,0 & 12,0 \\
1o Quartil & 20,5 & 23,0 & 22,0 \\
Mediana & 23,0 & 25,0 & 24,0 \\
Média (DP) & $23,5(6,3)$ & $27,1(5,2)$ & $25,3(6,0)$ \\
3o Quartil & 27,5 & 31,5 & 30,5 \\
Máximo & 34,0 & 35,0 & 35,0 \\
\hline
\end{tabular}

Pequena parte das crianças $(n=30 / 3,33 \%)$ apresentaram desempenho inferior e igual proporção $(n=30 /$ $3,33 \%)$ tiveram um desempenho médio inferior. Já a maioria ( $n=30 / 43,33 \%$ ) foram consideradas como muito superiores. $36,66 \%(n=30)$ foram classificadas tendo um desempenho superior. E de 30 participantes, $13,33 \%$ foram consideradas com um desempenho médio superior. São resultados consideravelmente melhores do que os dos restantes dos testes.

O subteste foi aplicado com o intuito de levantar suspeita de risco de dificuldade de aprendizagem de leitura. $\mathrm{Na}$ Tabela 3, é possível visualizar seus resultados, que levam em consideração a quantidade de itens repetidos corretamente pela criança.

Tabela 3: Média (pontos brutos) e desvios padrões comparados à média esperada por idade e desvios padrões respectivos para o subteste Dígitos de WISC-IV (com base no manual do teste).

\begin{tabular}{ccc}
\hline WISC & Média (DP) & $\begin{array}{c}\text { Média (DP) esperada por } \\
\text { Idade }\end{array}$ \\
\hline OD - 7 anos & $4,6(0,72)$ & $4,6(1,1)$ \\
OI - 7 anos & $2,6(0,48)$ & $2,7(1,1)$ \\
OD - 8 anos & $4,85(0,48)$ & $5(1,2)$ \\
OI - 8 anos & $2,73(0,58)$ & $3(0,9)$ \\
\hline
\end{tabular}

Nota: OD = Ordem Direta. OI = Ordem Inversa. 
Não ocorrem outliers no limite inferior, porém é possível notar um outlier com pontuação muito acima dos demais alunos, atingindo 16 pontos ponderados. Pontuações a partir de 7 para baixo são consideradas como inferiores pelo Manual do teste, fato este ocorrido com cinco alunos ( $n=30,16 \%$ ), cujas iniciais são: AAG, JSR, KLV, MHM e TAM. Observou-se que 25\% da amostra concentrou sua pontuação entre 4 e 8 pontos ponderados, correspondente ao primeiro quartil.

O teste BPA, utilizado para se medir a atenção geral por meio do desempenho das crianças em atenção alternada, concentrada e dividida, apresentou os resultados apresentados na Tabela 4.

Tabela 4: Médias (pontos brutos) e desvios padrões para o teste BPA.

\begin{tabular}{cccc}
\hline BPA & Média (DP) & $\begin{array}{c}\text { Média esperada por } \\
\text { Idade e DP }\end{array}$ & $\begin{array}{c}\text { Média esperada por } \\
\text { Escolaridade e DP }\end{array}$ \\
\hline AA & $54,36(13,43)$ & $44,34(16,67)$ & $58,63(25,37)$ \\
AC & $46,77(12,28)$ & $45,22(18,60)$ & $63,30(26,91)$ \\
AD & $44,56(17,79)$ & $35,48(26,33)$ & $44,34(30,94)$ \\
AG & $146,5(30,03)$ & $125,03(48,15)$ & $166,28(66,98)$ \\
\hline
\end{tabular}

Nota-se que, de uma maneira geral, as médias obtidas são superiores às médias esperadas por idade, propostas por Rueda (2013), contudo, inferiores quando se relacionam com o esperado para o ano de escolaridade. A justificativa para este resultado pode ser pela categorização ampla do quesito idade, que avalia com o mesmo crivo o desempenho de crianças de 6 a 10 anos de idade. 0 mesmo fato pode ser dito sobre o critério de escolaridade, onde os valores são avaliados de acordo com o período escolar no qual o indivíduo se encontra. No caso, os dados foram comparados com o item "Ensino Fundamental Regular", que abarca 9 anos de estudo, de acordo com a Lei no 11.274 (2006).

O BCPR foi aplicado com a intenção de poder levantar suspeitas de risco de dificuldades de leitura nas crianças. Ele qualificou $96,67 \%(n=30)$ do desempenho das crianças como dentro da média, e 3,33\% $(n=$ 30) como abaixo da média de memória fonológica, como observado na Tabela 7. A média de toda a amostra, sem divisão por idade, foi de 34,1 pontos brutos e desvio padrão de 4,36.

Tabela 7: Médias (pontos brutos) e desvios padrões para o teste BCPR.

\begin{tabular}{ccc}
\hline BCPR & Média (DP) & $\begin{array}{c}\text { Média esperada por } \\
\text { Idade (DP) }\end{array}$ \\
\hline 7 anos & $33,47(3,83)$ & $32,2(4,5)$ \\
8 anos & $34,66(2,93)$ & $35,3(3,0)$ \\
\hline
\end{tabular}

Não existem no Brasil normas para as tarefas de NSR. Os poucos estudos na área possuem amostras muito pequenas e/ou com grupos com grande amplitude de idade. Portanto o desempenho médio da amostra foi considerado como referência para a identificação dos outliers. Seguindo a literatura (ex., Cortez, Souza e Pinheiro 2019; Tannock et al. 2000) as tarefas de nomeação de letras e de números, foram consideradas para a identificação de crianças em risco de TEAp.

A Figura 1 mostra as médias (pontos interligados por traços) e os desvios padrões (barras verticais) do tempo em segundos de cada participante (cada um é representado por um ponto). Pode-se perceber que 
as médias em Letras e Números estão visualmente próximas, um pouco distantes da média de Cores e bastante distantes das médias de Objetos.

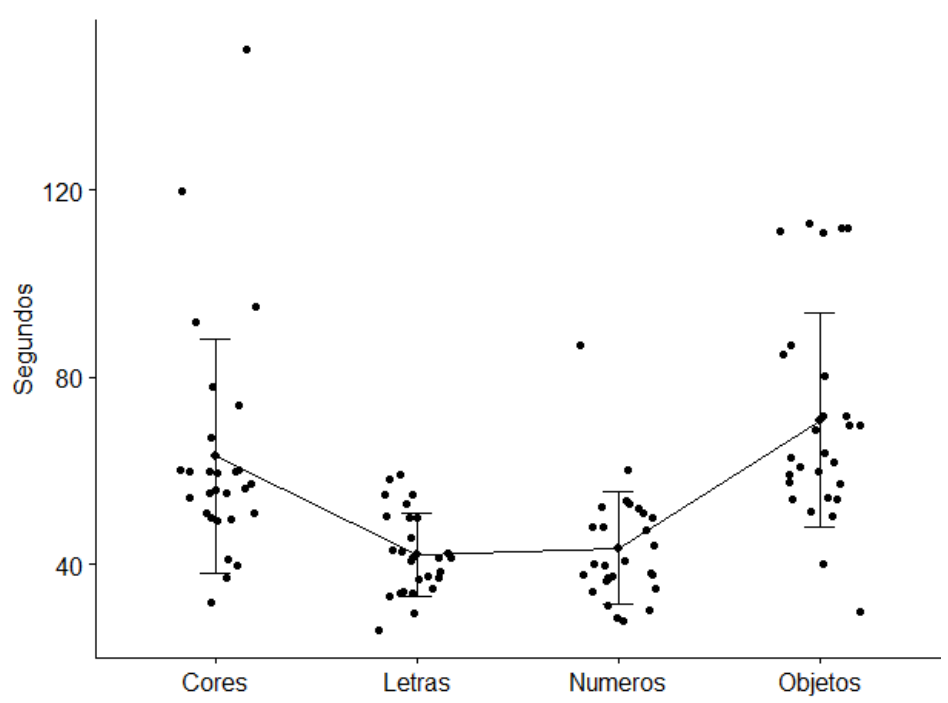

Figura 1: Desempenho da amostra completa para cada tarefa de NSR: médias interligadas horizontalmente, desvios padrões nas barras verticais.

A Tabela 8 apresenta a média e desvio padrão para a amostra para o teste TELCS, de acordo com Vilhena e Pinheiro (2020). O desempenho de 14 crianças está acima da média ( $n=30,46,66 \%)$, de 10 na média $(n=$ $30,33,33 \%$ ) e de 6 se abaixo da média (grupo de Risco) ( $n=30,20 \%)$.

Tabela 8: Médias (pontos brutos) e desvios padrões para o teste TELCS, considerando os dados referenciais do $2^{\circ}$ ano escolar.

\begin{tabular}{ccc}
\hline TELCS & Média (DP) observados & Média (DP) esperados \\
\hline 2 o ano & $11(5,28)$ & $9(6,4)$ \\
7 anos & $9,1(3,0)$ & $7,9(6)$ \\
8 anos & $13(6,66)$ & $13,1(7,4)$ \\
\hline
\end{tabular}

A Tabela 9 mostra a distribuição dos resultados em seus graus de eficiência, identificando a quantidade de alunos classificados em cada desempenho e sua porcentagem em relação ao todo da amostra.

Tabela 9: Distribuição da amostra segundo desempenho no TELCS, com número e porcentagem de alunos em cada categoria.

\begin{tabular}{ccc}
\hline TELCS & $\begin{array}{c}\text { Quantidade } \\
\text { de Alunos }\end{array}$ & Porcentagem \\
\hline Transtorno & 4 & 13,33 \\
Dificuldade & 2 & 6,66 \\
Média & 10 & 33,33 \\
Média Superior & 9 & 30 \\
Superior & 5 & 16,66 \\
\hline
\end{tabular}

Os alunos considerados "com transtorno" pelo TELCS são os de respectivas iniciais: AOG, CLF, KLV e MCA. 


\subsection{Análises correlacionais}

As correlações dentro do próprio domínio, sendo todas elas significativas. De maneira geral os subdomínios que compõem o IMTAP-Cog se correlacionam entre si. Apresentam uma gama de correlações consideradas medianas, com exceção dos subdomínios "Fundamentos" e "Tomada de Decisão", cujo índice de correlação foi o mais baixo $(r=0,371)$. Dentre as correlações moderadas merece destaque o índice encontrado entre "Recordação de Curto Prazo" e "Recordação de Longo Prazo" ( $r=0,709)$. O padrão de resultados obtidos indica que há uma certa consistência dos subdomínios entre si.

Contrário às expectativas não foram encontradas correlações significativas entre o IMTAP-Cog com nenhum dos testes escolhidos para prover a esse instrumento validade convergente, exceto com as MPCR, mas com um índice de correlação baixo $(r=0,336)$. O que significa que os dados não apresentam associação ou relação significativa que seja de interesse e relevância.

Confirmando as expectativas, não foram encontradas relações significativas entre o IMTAP-Cog o TELCS e entre o IMTAP-Cog e as tarefas de NSR, uma vez que os dois instrumentos (TELCS e a NSR) medem construtos diretamente relacionados ao bom desempenho em leitura.

\section{Discussão}

O objetivo geral da pesquisa, prover validade convergente para o IMTAP-Cog, não foi atingido. Isso pode significar que as atividades elaboradas nessa pesquisa para medir "Cognição" não tenham abrangido o mesmo construto dos instrumentos utilizados para medir funções cognitivas equivalentes, tais como diferentes tipos de atenção (avaliadas pelo BPA) e recordação de curto prazo (avaliada pelo subteste Dígitos do WISC e pelo BCPR). Esses resultados apontam para a necessidade de maior domínio do construto subjacentes às atividades propostas e sua articulação com as especificidades da área de Musicoterapia.

O teste Raven, por buscar analisar níveis de inteligência ligados ao raciocínio lógico e à capacidade de abstração, foi o único teste se correlacionou significativamente com o IMTAP-Cog. Embora o índice de correlação tenha sido baixo, existe pelo menos um indicativo de que a inteligência possa ser fator de importância dentro do contexto de um bom desempenho no IMTAP-Cog. O teste avalia o fator de inteligência chamado "Fator g", proposto por Spearman (1961), que, segundo esse pesquisador, representa uma junção de todas as habilidades cognitivas necessárias para a execução de uma tarefa, sendo o atributo que melhor qualifica a eficiência no desempenho intelectual.

Quanto à validação divergente, o TELCS, a única medida de reconhecimento de palavras escritas e de compreensão de leitura empregue nessa pesquisa, não se correlacionou com o IMTAP-Cog, que indica que os dois instrumentos, conforme a expectativa, testam construtos diferentes.

O IMTAP, o instrumento da área identificado como o mais avançado, apesar de ter introduzido a quantificação dos escores, falha por não se desvincular das limitações da área de Musicoterapia. Assim, mantém a flexibilidade na elaboração (ou escolha) das atividades e de procedimentos. Mostra inclusive, falta de clareza nas instruções desses procedimentos, o que causa confusão e dúvidas no entendimento dos enunciados das atividades, além da sensação de repetição de tópicos (quando toda a escala é aplicada), conforme relato dos musicoterapeutas do estudo de Salokivi (2012), por exemplo. Todas essas limitações, somadas à ausência de expectativas sobre o desempenho esperado com base em grupos de referência 
pautados em faixas etárias, tornam temerárias a utilização do instrumento em sua íntegra ou por domínio na pesquisa.

A liberdade de criação de atividades proporcionadas pelo IMTAP torna o seu conteúdo subjetivo e mutável, o que dificulta a sua comparação com as medidas que têm seus construtos bem definidos. Na realidade, em seu estado atual, o IMTAP oferece ao musicoterapeuta apenas um quadro de referência para a criação de suas próprias atividades. Então, no caso de uma validação, o que se estaria validando seria um conjunto de atividades específicas criadas por um musicoterapeuta específico, o que sinaliza a urgência de se estabelecer para cada domínio do protocolo um corpo de atividades definidas. Além desse problema conceitual, as instruções disponíveis sobre a aplicação do IMTAP carecem de uma sistematização mais rigorosa e específica. É possível que as confusões e dúvidas, percebidas inclusive pelos observadores ao criar e avaliar as atividades tenham advindo de uma falta de direcionamento para as necessidades mencionadas no protocolo. A falta de um norteio para a construção de atividades pode prejudicar o desenvolvimento da ação na prática, reduzindo as chances de comprometimento na observação final.

\section{Conclusão}

Mesmo com os avanços alcançados na última década, a Musicoterapia ainda é uma área carente de materiais, estudos e instrumentos, fato este justificável também pela jovialidade da área. A cada dia, musicoterapeutas lutam para alterar esta realidade que traz consequências injustas para todos envolvidos. Trabalhos como este reforçam a necessidade de mais pesquisas, desenvolvimentos e sistematizações dos instrumentos e procedimentos utilizados, para que um dia essa forma de terapia seja reconhecida como instrumento necessário e indispensável no trabalho de diagnósticos e tratamentos de indivíduos que tenham as mais variadas queixas e necessidades.

Em relação à presente pesquisa, as atividades desenvolvidas não alcançaram o êxito esperado. Percebe-se que o objetivo de se encontrar uma ponte entre um conhecido instrumento da área musicoterapêutica e instrumentos já muito utilizados pela psicologia para a deteç̧ão de Transtornos Específicos de Aprendizagem não foi atingido. Este fato se deve, em grande parte, aos enormes vácuos e desestruturações presentes no protocolo IMTAP. Para estudos futuros, é necessário que esforços sejam feitos para a criação de atividades definidas e testáveis, não só para o domínio "Cognição" da IMTAP, mas para todos os outros domínios do protocolo. Sugerimos também que se trabalhem com amostras maiores, em períodos longitudinais. Mais um incentivo de pesquisa seria o desenvolvimento de expectativas de desempenho com base em grupos de referência pautados em faixas etárias.

Dessa forma, uma sistematização dos procedimentos utilizados é necessária para avanços na área. Essa mudança não só propiciará que musicoterapeutas de todo o mundo possam dialogar entre si, mas que submetam seus procedimentos a testes experimentais para que possam mensurar de forma objetiva e eficiente suas contribuições para a ciência e aos seus públicos-alvo.

Embora tenha-se tentado fazer o mesmo aqui com o domínio Cognição, ao longo do trabalho percebeu-se que a liberdade de criação de atividades representa um desafio maior do que antecipado, o que abre a possibilidade de que o fracasso em se validar as atividades criadas e de se encontrar diferenças entre os grupos da pesquisa possam ter ocorrido em função de insuficiente exploração dos construtos da psicologia cognitiva. No entanto, no que se refere às crianças do grupo de risco, há de se considerar que, possivelmente seus desempenhos em relação ao grupo sem risco não tenham sido acentuados o suficiente para que 
pudessem obter resultados diferentes no IMTAP-Cog. Os estudos que sugerem a possibilidade de relação entre o processamento musical e a leitura encontrado na literatura são em pequeno número e em sua maioria conduzidas com crianças disléxicas.

Conclui-se assim que a flexibilidade na criação das atividades do IMTAP pode ser um fator complicador ao se considerar processos de validação. Ressalta-se a importância então de se haver instruções bem construídas para que o musicoterapeuta saiba a melhor forma de se construir uma atividade que avalie de forma eficaz os domínios e/ou subdomínios considerados.

\section{Referências}

Angelini, Arrigo Leonardo, Alves, Irai Cristina Boccato, Custódio, Eda Marconi, Duarte, Walquíria Fonseca, Duarte, e José Luciano Miranda. 1999. Manual matrizes progressivas coloridas de Raven: escala especial. Centro Editor de Testes e Pesquisas em Psicologia. São Paulo, SP, Brasil.

APA - American Psychiatric Association. 2013. Diagnostic and statistical manual of mental disorders: DSM5. 5. ed. Washington, D.C.: American Psychiatric Association. [edição brasileira: Manual diagnóstico e estatístico de transtornos mentais: DSM-5. 5. ed. Trad. Nascimento, Maria Inês Corrêa, Machado, Paulo Henrique, Garcez, Regina Machado, Pizzato, Régis, Rosa, Sandra Maria Mallmann. Porto Alegre: Artmed, 2014].

Barnett, Susan, e Stephen Ceci. 2002. When and where do we apply what we learn? A taxonomy for far transfer. Psychological bulletin, 128 (4): 612-637.

Baxter, Holly Tuesday, Berghofer, Julie Allis, MacEwan, Lesa, Nelson, Judy, Peters, Kasi, e Penny Roberts. 2007. The Individualized Music Therapy Assessment Profile: IMTAP. London: Jessica Kingsley Publishers.

Benson, Nancy, Lovett, Maureen, e Carolyn Kroeber. 1997. Training and transfer-of-learning effects in disabled and normal readers: evidence of specific deficits. Journal of experimental child psychology, 64 (3): 343-366.

Bruscia, Kenneth. 2016. Definindo Musicoterapia. 3a edição. Barcelona Publishers.

Cogo-Moreira, Hugo, Andriolo, Régis, Mari, Jair, e Latife Yazigi. 2009. Musical training for improving cognitive and motor development in children with reading difficulties. Cochrane Database of Systematic Reviews Issue, São Paulo, Brasil.

Coluci, Marina Zambom Orpinelli, Alexandre, Neusa Maria Costa, e Daniela Milani. 2015. Construção de instrumentos de medida na área da saúde. Ciência \& Saúde Coletiva, 20, 925-936.

Cortez, Marilene Tavares, de Souza, Luciana Karine de, Pinheiro, e Ângela Maria Vieira. 2019. É mesmo (só) Transtorno de Déficit de Atenção/Hiperatividade (TDAH)? Avaliando TDAH e encontrando Dislexia. Psico, 50(3), 29924.

Denckla, Martha, e Rita Rudel. 1974. Rapid "Automatized" Naming of pictured objects, colors, letters and numbers by normal children. In Cortex, 10, 186-202.

Deschamps, Eduardo. 2017. Base Nacional Curricular Comum (BNCC). Resolução CNE/CP № 2, de 22 de dezembro. Informações editoriais: Conselho Nacional de Educação do Ministério da Educação do Governo Federal da República Federativa do Brasil. Recuperado em 10 de setembro de 2019, de http://basenacionalcomum.mec.gov.br/images/historico/RESOLUCAOCNE_CP222DEDEZEMBRODE20 17.pdf 
Fisher, Douglas. 2001. Early language learning with and without music. Reading Horizons, 42(1), 40-49.

Forgeard, Marie, Schalaug, Gottfried, Norton, Andrea, Rosam, Camilla, Iyengar, Udita, e Ellen Winner. 2008. The relation between music and phonological processing in normal-reading children and children with dyslexia. University of California Press., 25, No 4. 383-390.

Foxton, Jessica, Talcott, Joel, Witton, Caroline, Brace, Hal, McIntyre, Fiona, e Timothy Griffiths. 2003. Reading skills are related to global, but not local, acoustic pattern perception. Nature Neuroscience. 6(4): 343344.

Gordon, Reyna, Fehd, Hilda, e Bruce McCandliss. 2015. Does music training enhance literacy skills? A metaanalysis. Frontiers in Psychology, 6, 1777.

Gregory, Dianne. 2000. Test instruments used by Journal of Music Therapy authors from 1984-1997. Journal Music Ther. Summer, 37(2):79-94. PubMed PMID: 10932123.

Habib, Michael, Lardy, Chloé, Desiles, Tristan, Commeiras, Céline, Chobert, Julie, e Mireille Besson. 2016. Music and Dyslexia: A New Musical Training Method to Improve Reading and Related Disorders. Frontiers in Psychology, 7, article 26.

Jaarsma, Birgitt, Ruijssenaars, Aloysius, e Willem van den Broeck. 1998. Dyslexia and Learning Musical Notation: A pilot study. Springer, 48.137-154.

Kilgour, Andrea, Jakobson, Lorna, e Lola Cuddy. 2000. Music training and rate of presentation as mediators of text and song recall. Memory \& cognition. 28 (5): 700-710.

Loureiro, Cybelle Maria Veiga, Rosário, e Verônica Magalhães. 2017. Técnicas da Musicoterapia Neurológica. Apostila com a tradução das principais técnicas da Musicoterapia Neurológica de acordo com Thaut (2008) e Thaut \& Hoemberg (2014). [Apostila da disciplina de Musicoterapia: lesado motor] Belo Horizonte, Brasil.

Lundetræ, Kjersti, e Jenny Thomson. 2017. Rhythm production at school entry as a predictor of poor reading and spelling at the end of first grade. Read Writ. 31: 215-237.

Montello, Louise, e Edgar Coons. 1998. Effects of active versus passive group music therapy on preadolescents with emotional, learning, and behavioral disorders. Journal of Music therapy, 35(1), 4967.

Moreno, Sylvain, Marques, Carlos, Santos, Andreia, Santos, Manuela, Castro, São Luís, e Mireille Besson. 2009. Musical training influences linguistic abilities in 8-year-old children: more evidence for brain plasticity. Cereb Cortex. 19(3):712-723.

O’Brien, Kate. 2004. Adaptating Music for Students with Dyslexia. Music Educator Journal, 90, n 5, 27-31.

Peretz, Isabelle, Vuvan, Dominique, Lagrois, Marie-Élaine, e Jorge Armony. 2015. Neural overlap in processing music and speech. Philosophical Transactions of the Royal Society of London. Series B, Biological sciences, 370, 20140090.

Pérez, María Alegría Vásquez. 2015. Terapia alternativa para la dislexia Musicoterapia. Bachelor's thesis, Quito: USFQ.

Pinheiro, Ângela Maria Viera, e Leonor Scliar-Cabral. 2017. Dislexia: causas e consequências. Belo Horizonte: Editora UFMG.

Raven, John Carlyle. 1949. Progressive matrices (1947), sets A, Ab, B: board and book forms. London: Lewis. 
Rueda, Fabián Javier Marín. 2013. Bateria Psicológica para Avaliação da Atenção - BPA. São Paulo: Vetor.

Salokivi, Maija. 2012. The individualized music therapy assessment profile as an initial assessment tool of social emotional functioning.

Santos, Flávia Heloísa, e Orlando Francisco Amodeu Bueno. 2003. Validation of the Brazilian Children's Test of Pseudoword Repetition in Portuguese Speakers Aged 4 to 10 Years. Braz J Med Biol Res 36(11), 15331547.

Schlaug, Gottfried, Norton, Andrea, Overy, Katie, e Ellen Winner. 2005. The Neurosciences and Music II: From Perception to Performance. Ann NY Acad Sci, 1060:219-230.

Silva, Alexandre Mauat da. (2012). Tradução para o Português Brasileiro e Validação da Escala Individualized Music Therapy Assessment Profile (IMTAP) Para Uso no Brasil. Dissertação para obtenção do título de Mestre do Programa de Pós-Graduação em Saúde da Criança e do Adolescente da Universidade Federal do Rio Grande do Sul, Porto Alegre, Brasil.

Silva, Alexandre Mauat da, Gattino, Gustavo Schultze, Araujo, Gustavo Andrade, Mariath, Luiza Monteavaro, Riesgo, Rudimar dos Santos, e Lavinia Schuler-Faccini. 2013. Tradução para o Português Brasileiro e Validação da Escala Individualized Music Therapy Assessment Profile (IMTAP) para uso no Brasil. Revista Brasileira de Musicoterapia. Ano XV, no14. 67-80.

Spearman, Charles. (1961). "General Intelligence" Objectively Determined and Measured. In J. J. Jenkins \& D. G. Paterson (Eds.), Studies in individual differences: The search for intelligence (p. 59-73). AppletonCentury-Crofts.

Tannock, Rosemary, Martinussen, Rhonda, e Jan Frijters. 2000. Naming speed performance and stimulant effects indicate effortful, semantic processing deficits in attention-deficit/hyperactivity disorder. Journal of abnormal child psychology, 28(3), 237-252.

Thaut, Michael. 2008. Rhythm, Music, and the Brain: Scientific Foundation and Clinical Applications. New York and London: Routledge Taylor \& Francis Group.

Thaut, Michael, e Volker Hoemberg. 2014. Handbook of neurologic music therapy. Oxford University Press (UK).

Thaut, Michael, Mclntosh, Gerald, e Volker Hoemberg. 2015. Neurobiological foundations of neurologic music therapy: rhythmic entrainment and the motor system. Frontiers in psychology, 5, 1185.

Thompson, William, Schellenberg, Glenn, e Gabriela Husain. 2004. Decoding speech prosody: do music lessons help? Emotion. 4(1): 46-64.

Urbina, Susana. 2004. Essentials in Validity. Hoboken: Essentials of psychological testing.

Vilhena, Douglas Araújo, Sucena, Ana, Castro, São Luís, Pinheiro, e Ângela Maria Vieira. 2016. Reading TestSentence Comprehension: An adapted version of Lobrot's Lecture 3 Test for Brazilian Portuguese. Dyslexia, 22(1),47-63. doi: 10.1002/dys.1521.

Vilhena, Douglas Araújo, Pinheiro, e Ângela Maria Vieira. 2020. Reliability, Validity and Standardization of the Reading Test: Sentence Comprehension [Fidedignidade, Validade e Normatização do Teste de Leitura: Compreensão de Sentenças]. Psicologia: Teoria e Pesquisa, no prelo.

Wechsler, David. 2013. Escala de Inteligência Wechsler para Crianças: WISC-IV. Manual de instruções para aplicação e avaliação. Adaptação e Padronização Brasileira: Rueda, F. J. M., Noronha, A. P. P., Sisto, F. F., Santos, A. A. A., \& Castro, N. R. C. 4ed. São Paulo: Casa do Psicólogo. 